\title{
26
}

\section{Conclusions and Future Policies for Meeting the Sustainable Development Goals}

\author{
Ademola A. Adenle, Marian R. Chertow, Ellen H.M. Moors, \\ and David J. Pannell
}

\section{Introduction}

Science, technology, and innovation (STI) policy interventions are key to addressing the 17 sustainable development goals (SDGs) leading to increased productivity, sustainable growth, and prosperity around the world, and especially in developing regions. The SDGs are more tightly and explicitly related to STI solutions than were the millennium development goals (MDGs). An illustration of this is the establishment in 2015 of the technology facilitation mechanism (TFM), which aims to facilitate technology transfer to address the gap between developed and developing countries in STI capacity and to coordinate a multistakeholder forum on sustainable development, including SDGs. Beyond technology transfer, delivering STI solutions will require the bolstering of legislative support, business commitment, coordination with governments and civil society organizations of all types, and the strengthening of STI strategies at the country level. There is a consensus within the international community that developing strong STI capacity at the national level is critical to building thriving economies and societies and finding long-term solutions to national and global problems (UN 2016).

This book has provided insight into actual and potential STI contributions for the achievement of SDGs, covering a wide range of STI initiatives across numerous countries and sectors. It has explored the interconnections between the SDGs and STI capacity, often with a focus on the implementation of SDG projects. This book has emphasized intersectoral collaboration and interdisciplinary approaches to addressing the challenges of SDGs across the themes of environment and energy, health, and agriculture. The content of the book includes case studies, experiences, and empirical analyses, identifying some of the most significant roles that STI solutions can play in meeting the socioeconomic and environmental challenges spelled out in the SDG targets.

The chapters have covered a broad range of emerging issues and factors influencing the development of effective STI policies, and how such policies may contribute to 
or undermine the achievement of SDGs. In this conclusion we review contributions from all of the chapters, drawing out common themes, differences, and key lessons. Conclusions are identified for each theme and we finish with a set of recommendations based on the insights provided in the earlier chapters.

Previous studies indicate the potential role of STI in tackling global challenges including poverty, food insecurity, diseases, and climate change, yet in many developing countries, little attention is paid to harnessing STI in addressing these problems (Adenle et al. 2015; UN 2014; World Bank 2008). The global development agenda, including MDGs, often underemphasized the potential for STI contributions, resulting in impacts that fall short of their potential. Contributors to this underperformance have included limited investment in research and development $(\mathrm{R} \& \mathrm{D})$, poor protection of intellectual property rights (IPRs), insufficient knowledge infrastructure, lack of institutional support for development and diffusion of STI solutions, and lack of adequate regulatory instruments that could create incentives for investment in STI solutions. The experiences and evidence presented in this book illustrate how a failure to provide the institutions and resources needed to build STI capacity, and a failure of key actors to engage synergistically, can be serious impediments to development.

Prior to this book, there had been relatively little interdisciplinary writing about STI and the SDGs. The interdisciplinary approach used here has cast light on a number of questions, including: How can STI capacity be enhanced and harnessed at the national level to meet specific SDG targets? What can be done to improve international STI frameworks that can enhance the implementation of SDGs? How can we reliably test new ideas for harnessing STI to deliver SDGs?

The aims of this book are to examine the roles that STI can play in contributing to the SDGs, to understand factors aiding or impeding successful application of STI, taking into account the interlinkages among SDGs, and to investigate the interaction of STI and SDGs in a broad variety of settings and geographies.

\section{Key Messages}

Theme I of the book (environment and energy) offers the following set of findings to assist with achieving SDGs: 1) With respect to the four chapters on energy, renewable technologies present strong opportunities for inclusive social and economic development and can also contribute to addressing environmental problems, particularly those related to climate change and the implementation of the 2015 Paris Agreement; 2) Chapters 3 to 6, which focus on solar energy, laid out numerous conclusions that have emerged from research over the last 15 years, emphasizing specific sociological and governance conditions that slow the pace of diffusion, thereby inhibiting effective responses to climate change (SDG13) and biodiversity loss (SDG15). Solar energy is a gateway not only to increased human development, but also to significant changes in the current system of production and consumption across different sectors and industries, offering a sustainable development path based on a system 
of green innovation; 3) environment and energy outcomes can benefit from policy measures that target low-carbon innovation and market-development mechanisms while supporting national STI capacity building (e.g., research institutes, universities, R\&D investment, and IPR systems); and 4) assessment tools such as input-output analysis (IOA) and life-cycle assessment (LCA) enable comparisons of different ways to achieve similar goals in environmental terms so that less harmful solutions can be identified and selected.

With respect to barriers to energy development, chapter 3 (Timilsima and Shah) highlights a wide range of problems facing the renewable energy sector, characterized by the presence of market, capacity, and regulatory barriers in the developing world. The authors make the case that these barriers must be overcome so that SDG7 and other relevant SDGs can benefit from the advantages of renewable energy technologies. Chapter 4 (Adenle) underscores the need to develop robust renewable energy policy that encourages investment in R\&D for solar energy programs in view of limited institutional capacity across African countries. Overcoming barriers that limit the abilities of developing countries to introduce renewable energy technologies successfully enables participation in competitive market-based economies.

As described in chapter 5 (Schmidt et al.), regulatory challenges in Cambodia, Laos, and Indonesia remain important problems limiting the design, installation, and operation of renewable-energy micro-grids, which could undermine achievements within SDG7. Similarly, chapter 6 (Kemp et al.) contrasts the enormous growth of installed solar capacity in India based on auctions and other market-oriented approaches with China's reliance on the governance role of state institutions and programs for successfully harnessing STI to meet SDG7.

With respect to the environmental aspects of Theme 1, environment and energy, we report findings at the human/environment interface based on policies and programs in the natural environment (chapters 2 and 7) and on tools and technologies in the built environment (chapters 8 to 10). As with renewable energy, the environmental policy themes reflect that national and international level policies can be effective, but they require significant supporting resources.

Chapter 2 (Stevens) examines a target of SDG15 to halt biodiversity loss. By examining the role of innovation in biodiversity conservation, the author finds that the management pathway developed through the use of action plans advanced under the Convention on Biological Diversity can be used effectively at the international level but depends on a great deal of planning and coordination now and to the SDG deadline of 2030. Chapter 7 (Machado and Young) scrutinize the R\&D needed for environmental conservation and sustainable use of natural resources in Brazil, but rather than placing as much attention on the interplay of institutions as Stevens did, the chapter investigates the financial resources available to meet the country's SDGs; a large shortfall is found by 2030. This leads the authors to discuss improvements needed for STI funding policy and to consider making SDG targets mandatory for determining resource allocation. 
Chapter 8 (Chertow et al.) introduces several tools and strategies from the field of industrial ecology for determining how SDG-related activities and technologies can be assessed to determine their environmental and social performance. The authors report how these tools can identify trade-offs of different alternatives such as impacts of public transport versus private vehicles applicable to SDG9 on cities or where in a food supply chain the highest amounts of food waste are generated useful for SDG2 on hunger.

Delving further into technology, the case of automated vehicles discussed in chapter 9 (Wang and Oster) revolves around the call in SDG12 to make cities inclusive, safe, resilient, and sustainable. The authors show, for example, how providing low-speed driverless shuttles for city residents can increase safety and inclusivity by coordinating with public mass transit rather than competing with it. But of course technology also has its limits, which can lessen the impact of SDGs through rebound effects described in chapter 10 (Font Vivanco and Makov). The irony of rebound effects is that an environmentally sound technology may encourage consumers to use more of a given product than when the same function was fulfilled by the product when it had less desirable characteristics. The authors find, therefore, that rebound effects can easily serve as hidden barriers for achieving SDGs by offsetting some of the positive effects.

Theme II (health) describes scientific advances and the role of innovative technologies in addressing global health problems. Here, the authors emphasize the following key points toward achieving SDG3 (healthy lives) and other relevant SDGs: 1) harnessing STI is critical for the delivery of effective and low-cost health technologies; 2) removing regulatory barriers (including the logjam around international property rights) can facilitate access to vaccines against emerging and neglected infectious diseases in low-income regions; 3) increased availability, affordability, accessibility, and acceptability of anti-malaria drug development can facilitate production, diffusion, and deployment of new drugs in affected regions; 4) deployment of analytical tools such as development-focused health technology assessment (HTA) can help decision-makers to prioritize and make new health technologies and innovations more accessible both in developing and developed countries; 5) recent progress of digital health can be important in improving the quality of healthcare; 6) support for long-term transformations of urban sanitation services is important in low- and middle-income countries (LMICs) to reach the health-related SDGs; and 7) health innovations needed to deliver SDGs should be pursued in responsible and responsive ways.

The chapters in the health theme describe how STI are conceptualized in the SDGs to ensure healthy lives and to promote well-being for all (SDG3), and also how they are assumed to tackle health inequalities (SDG10) and to assure availability of water and sanitation (SDG6) (e.g., chapters 11, 13, 15, 16, and 17). By exploring cases in the field of vaccine innovations, anti-malarial drug developments, point-of-care diagnostics, cookstoves, urban sanitation projects, and animal-source foods, these chapters show that global health issues should be defined as multidimensional, multiactor 
problems. In contrast to the MDGs, the SDGs entail a global and equitable approach to these global health issues.

Chapter 11 (Possas et al.) shows that a number of the SDGs are related to vaccination problems. They elaborate on the specific technological and regulatory gaps in developing countries that inhibit the delivery of health-related SDGs, such as limited commercial interest in neglected diseases and limited access to patented vaccines. Adequate STI governance strategies can overcome many of these challenges and increase access to essential medicines and vaccines. An example is the Decade of Vaccines initiative, described in chapter 11 . Vaccines developed by novel STI strategies require adaptations at a systems level, such as accompanying immunization services to reach the poorest areas with mobile units and adequate capacity-building and infrastructure.

Chapter 13 (de Haan and Moors) shows the importance of using an availability, affordability, accessibility, and acceptability framework to analyze the development and diffusion of anti-malarial medicines in light of the SDG goals, particularly the SDG3 targets. This chapter shows that the malaria burden in LMICs has been strongly reduced due to improved access to effective interventions for malaria prevention, diagnosis, and treatment. According to the authors, resistance to artemisinin and associated drugs has developed and is currently spreading. Therefore, innovative R\&D approaches are required to restore anti-malarial efficacy and to circumvent the spread of resistance to other areas, which could have a huge public-health impact.

Chapter 14 (Poon et al.) shows the interesting relationship between emerging digitalization in health and its relation to various SDGs. The authors use a fourdimensional perspective, zooming in on translation, education, transformation, and technology to examine the progress of digital health in China and its potential to improve the quality and delivery of healthcare to enhance health at all ages (SDG3) and the way health information is shared through new value chains in the health system. Part of the authors' emphasis is that digital health literacy and expansion of complementary skills among health professionals and patients will become more important to ensure inclusive and equitable quality education and to promote lifelong learning, thereby contributing to the achievement of targets for SDG4 (equitable quality education). Additionally, transforming health service supply in LMICs through digital technology will enable better access to services, including in rural areas, which often have poorer access than do urban areas. Also, progress in digital health can also reduce inequality, thereby contributing to SDG10 targets.

Chapter 16 (van Welie and Truffer) shows the potential of STI to address urban sanitation problems, an important issue as progress toward the SDG on water and sanitation (SDG6) is very slow and problems are especially persistent for cities in the Global South. Such a complex problem demands a transformation of urban sanitation services and infrastructure in view of challenges identified by the authors. Some of these challenges include limited participation of local communities, lack of adequate public services, and lack of awareness. The authors argue that STI efforts should focus on improving alignment among various sanitation-service regimes in the city 
through making utility services work better in informal settlements and improving collaboration and participation of relevant stakeholders to scale on-site sanitation innovations.

Chapter 17 (de Bruyn et al.) focuses on STI to promote the value of animal-source foods for meeting health-related SDGs in resource-poor regions and other settings. A holistic approach is needed, targeting stakeholder engagement for promotion of animal-source foods to meet health-related SDGs. According to these authors, the use of mobile technologies, for example, can support important information for livestock production. The authors also mention that gender-sensitive and culturally sensitive communication (incorporating traditional beliefs) can encourage participation in nutrition programs in resource-poor settings. These insights may contribute to achievement of SDG3 targets.

Chapter 12 (Bouttell et al.) gives an overview of the role of development-focused HTA to achieve the health-related SDGs. LMICs are now beginning to develop HTA and apply it in their healthcare decision-making. This chapter shows how a development-focused HTA analysis of low-cost point-of-care diagnostics can potentially improve the return on investment in new technologies by improving the efficiency of research prioritization and development processes while ensuring that the needs of vulnerable populations are met. Furthermore, chapter 15 (Engel et al.) shows that taking a responsible and responsive lens on STI is very important for inclusive SDG policies taking a multiactor perspective of different stakeholder settings involved, and their perceptions and needs.

The point-of-care approach highlighted in chapters 12 and 15 underscores the importance of frugal innovation that can provide access to affordable and cost-effective diagnosis and treatment for low-income and poor populations. Frugal innovation is increasingly seen as a potential solution to overcome challenges such as access to water, sanitation, and energy security for those who live in resource-constrained communities around the world. In chapter 16, van Welie and Truffer mention that a pro-poor initiative for on-site sanitation services has been developed by social enterprises in Kenya to address sanitation problems. This is consistent with ideas laid out by Adenle in chapter 4 (Theme I) that government policy should support R\&D efforts that support production and redesign of solar products, thereby making services and systems more affordable for the rural poor in Africa. With these measures being put in place, a targeted policy approach to support frugal innovation can help deliver some SDG targets around the world.

Taken together, the chapters in this theme highlight that health and STI policymakers should demand SDG-inclusive innovative health technologies. National STI policy should focus on responsible health technology development in context and should create STIs that are responsive and flexible for a range of situations. Rather than relying on health technology transfer from developed to developing countries, however, the SDGs require us to go deeper as described in this theme. More holistic approaches, given the high potential for unintended consequences, also address the 
impacts of STI with respect to reducing injustice and inequality and increasing health and well-being on a global basis.

Key messages emerging from Theme III (agriculture) include the following: 1) agricultural R\&D is critical to ensure a flow of new technologies and innovations that allow farmers and others in agricultural supply chains to address SDGs; 2) there are concerns about the concentration into a few hands of intellectual property rights for the seeds of improved agricultural crop varieties, raising the risk of monopolistic pricing strategies; 3 ) sophisticated packages of technologies and practices designed and promoted to farmers can help advance SDGs, provided that the packages are well designed to meet farmers local needs and to fit with their context and challenges; 4) there is an increasing need for R\&D to strengthen the emphasis on sustainability, not just productivity, when addressing the needs for farmers in developing countries; 5) well-designed agricultural innovations and technologies can contribute simultaneously to multiple SDGs, commonly including SDG1 (end poverty), SDG2 (end hunger), SDG8 (economic growth, employment, and decent work), and SDG13 (combat climate change) and sometimes others; 6) policy settings regulating technologies in developing countries should reflect needs and risks in those countries rather than in developed countries; 7) effective systems for agricultural extension (including awareness-raising measures and technical support) are needed to accelerate the spread of improved agricultural practices and technologies in developing countries; and 8) leverage points for delivery of SDGs may be along the food supply chain, rather than on farms.

Agricultural extension is one approach to scaling up the adoption of agricultural innovations that could contribute to delivery of SDGs. Others include farmer field schools, innovation platforms, subsidies, taxes, and marketing. Chapter 25 (Wigboldus et al.) focuses on the issue of scaling up, identifying it as a key issue for policymakers and development agencies to address. They provide a framework for thinking about and planning the scaling process, encompassing four key issues, in brief: the consequences of scaling, both societal needs and societal concerns, the needs for analysis and collaboration, and reflexive and adaptive management. These elements can contribute to defining a theory of scaling that the authors propose should form part of any theory of change. The approach provided in this chapter is relevant across all agricultural development issues.

Several chapters focus on particular agricultural technologies, practices, or packages. Chapter 18 (Harpankar) explored a range of technologies related to the nitrogen fertility of crops, and discussed their potential to contribute to various SDGs. A particular challenge with nitrogen fertility is providing sufficient nitrogen to achieve high crop yields (supporting SDGs 1, 2, and 8) while avoiding nitrogen pollution in water bodies (affecting SDG6 and SDG14). The technological options range from traditional (legume rotations) to high-tech (biotechnology) and all are seen as having potential. However, the barriers facing smallholder farmers in developing countries when considering adoption of these practices are substantial, including issues of affordability, technical expertise, infrastructure, and market access. 
Chapter 19 (Rola-Rubzen et al.) and chapter 21 (Mwongera et al.) both dealt with complex packages of agricultural practices and technologies in developing counties. Both considered developing regions where traditional agricultural practices with low productivity are still widely used. Both packages are intended to contribute to the resilience of farmers, particularly in the face of climate change. Rola-Robzen et al. emphasized the importance of using effective strategies for agricultural extension, awareness raising, capacity-building, and technical advice. Mwongera et al. prioritized various practices in different agricultural regions based on farmers' preferences, and found that the top priorities contributed to food security and livelihoods. Understanding farmers' preferences and needs is an important element of designing effective approaches to agricultural extension.

The important role of policy and governance is highlighted in several chapters in the agriculture section. Adenle et al. (in chapter 20) observes that policy constraints on the use of genetically modified organisms (GMOs) or genetic modification (GM) technology in various developing countries are inhibiting the achievement of various SDGs. They question the application of a highly risk-averse policy approach, similar to that applied in Europe, in countries where the cost in lost development opportunities is much higher than in the wealthy countries of Europe. Wedig (in chapter 23) is also concerned with existing policy and governance settings, this time in the context of small-scale fishers on Lake Victoria in Africa. Lake Victoria supports large numbers of small-scale fishers, as well as larger commercial fishing companies. Wedig argues that existing governance favors the larger companies, with potential negative consequences for SDG1 (end poverty), SDG2 (end hunger), and SDG14 (sustainable marine resources). She argues for a new transformation governance based on a threefold structure: increasing eco-efficiency, redistributing access to natural resources, and recognizing eco-sufficiency as a guiding principle.

Chapter 22 (Flocco) examined the soybean production complex in Brazil, exploring various ways to make the system contribute better toward various SDGs. STI are central to many of the approaches discussed, including contributions from the private sector. Reinforcing the message of Adenle et al. in chapter 20, genetically modified crops have played major roles in advancing the development of Brazilian agriculture, including making it more sustainable through reductions in use of pesticides and use of less toxic herbicides. Flocco notes that there have been concerns about GM technology in Brazil too, although they tend to be more about monopoly power and mono-cropping rather than unsubstantiated concerns about food safety.

As in other sectors, there can be trade-offs between SDGs in agriculture. An example is that the success of GM soybeans in Brazil led to pressure for deforestation to increase the area of land used for soybean production (chapter 22). An interesting initiative to counter this was the Soy Moratorium, which substantially reduced clearing, in part by facilitating market access for soybeans not associated with deforestation (or slave labor or threats to indigenous lands).

Flocco also highlighted that there are many stakeholders of many different types who impinge on the performance and impacts of an agricultural production 
complex. For Brazilian soybeans she identified farmers, local communities, private sector, commerce, transport, trading institutions, banks and financing institutions, producer's associations and commercial chambers, government, certifying institutions, multistakeholder dialogue tables, nongovernmental organizations, academic and research institutions, and consumers. This highlights the importance of a broadbased multipronged approach to the delivery of SDGs, rather than a narrow focus on one group. STI has contributions to make in all parts of this complex system.

Finally, Dentoni et al. (chapter 24) present value network analysis (VNA) as a diagnostic tool for analyzing business models that are being reorganized. They illustrate its application to the agricultural commodity exchange (ACE) in Malawi, a business model with complex objectives: to increase value-chain efficiency while fostering food security and reducing rural poverty and marginalization. In this case study, the approach helps to identify options for building cross-sector partnerships and coordinating actions (including STI actions) to tackle relevant SDG targets.

\section{The Importance of Systems Approaches}

Just as the SDGs have evolved to be cross-cutting and interdisciplinary, STI proposals, too, are seen in many of the chapters to have embraced a broader, systems approach. We believe that this perspective can facilitate implementation of the 2030 Agenda, including the SDGs. An example from each theme illustrates this point.

In the environment and energy theme, chapter 9 on automated vehicles analyzes this new technology from the perspective of being embedded in a pre-existing city. As game-changing as the technology appears to be, the importance of numerous other social, political, and infrastructural considerations are critical aspects of whether this is a viable solution for meeting SDGs and how these issues affect the overall adoption timeline for this innovation.

From the health theme, chapter 16 presents a systemic perspective on the global sanitation challenge. This chapter highlights the potential of STI to solve urban sanitation challenges, which are serious and persistent in many cities of LMICs. Using a sociotechnical systems perspective, the authors analyze the interplay between technologies, infrastructure and associated actor networks, regulations, sanitation providers, and user practices regarding sanitation. The authors specifically mention that various system weaknesses related to actors, networks, and institutions represent significant challenges to the implementation of improved sanitation systems. Therefore, STI efforts should focus on improving alignment and collaboration with the key stakeholders to scale on-site sanitation innovations to contribute to the achievement of SDG6 targets.

Chapter 19, from the agriculture theme, analyzes integrated crop management (ICM) in the context of the multifaceted constraints faced by farmers in Timor Leste. ICM is a production system that combines multiple components and delivers to multiple SDGs. Despite evidence of significant benefits from ICM, evidence shows that 
adoption of the system by farmers has been partial and biased. One important factor is that technical advice and training has been targeted to male farmers; not surprisingly, adoption of the system by female farmers is substantially lower. Another observation, one that is common in studies of adoption of agricultural innovations, is that adoption is more likely on larger farms. This is explicable in terms of the broader gains that larger farmers stand to make, and their greater capacity to invest the resources needed to learn about and implement the new innovation. Understanding this social, technical, and economic system provides insights into the sorts of initiatives needed to deliver development outcomes more effectively and more equitably.

In summary, the benefits of systems approaches are readily apparent. National STI policies can help to foster systems and networks that include governments, the private sector, community leaders, and international organizations.

\section{Policy Recommendations}

Since the SDGs were adopted in 2015, donors have provided billions of dollars for various related initiatives, and citizens show increasing awareness of the global goals. From a policy perspective, the most pressing issues discussed in this book relate to the need to improve the international STI framework and to encourage countries to increase their STI capacity so that they can better address sustainable development challenges. With higher recognition and visibility of SDGs, intergovernmental organizations, national governments, and donors (from the public and private sectors, charitable organizations, multilateral agencies, and others) can direct more resources and attention to solving institutional, market, and political issues that are impeding STI progress for sustainable development.

Such an STI approach resonates with the European Union's increasingly popular mission-oriented approach to policies. Mazzucato (2018) stresses that innovation programs should be bold, inspirational, and of wide societal relevance to engage the public and attract investment. These programs should be targeted, measurable, and time-bound enough to allow effective monitoring and evaluation. Further, Mazzucato argues that missions should be formulated ambitiously (high-risk but realistically feasible, centered on STI innovation activities) (Mazzucato 2018). In this light, we offer the following suggestions and recommendations:

1. That national STI policies adopt strategies that are market-driven (where relevant), stimulate socioeconomic growth and well-being, promote cooperation, and provide protection of property rights but also access by the poor to beneficial IP.

2. That country-level governments advance national innovation systems specifically for developing strong STI capacity that can foster sustainable development. There are a range of issues to be addressed affecting the capacity of STI to contribute to SDGs including: educational curricula; educational and 
research infrastructure; public funding for R\&D; and translation, outreach, and extension to deliver the benefits of STI to business and the community.

3. That broad and equitable community benefits be seen as foundational to STI programs and that the governance systems aim to support efficient institutions, strong legal frameworks, transparent decision-making, and provision of incentives, including business incentives, that acknowledge and encourage STI for SDGs.

4. That STI policy foster initiatives designed to deliver frugal innovation-the process of reducing the cost and complexity of a technology to make it more accessible. Particularly in developing countries, frugal innovation can stimulate social entrepreneurship, socioeconomic empowerment, and sustainable development by addressing food insecurity, energy problems, and health and well-being at the grassroots level.

5. That STI policy bodies take into account the dimensions of availability, affordability, accessibility, and acceptability when analyzing emerging STI solutions to health and sustainability problems.

6. That $\mathrm{R} \& \mathrm{D}$ around lower cost renewable energy technologies continue to be a mainstay of international development, contributing to energy access and achievement of inclusive social and economic development. Such R\&D can be complemented by local training and support designed to increase employment and human development.

7. That there be encouragement for digital health STI policies to transform health services in LMICs and to increase digital health literacy and skills of health professionals and patients. This would help to achieve better access to health services, reduce education inequality, and increase lifelong learning, contributing to universal health, sustainable education, and national equality.

8. That STI strategies be better contextualized by taking an inclusive multiactor perspective into account when considering the pathways to SDGs. This implies proactively addressing in STI strategies the specific needs, responsibilities, and perceptions of involved stakeholders and the particular settings in which they are operating, by applying a sociotechnical systems perspective that enables analysis of the interplay between technologies, infrastructures, and their associated actor networks, institutions, and user practices.

9. That the role of development-focused HTA to achieve the health-related SDGs be emphasized in STI policy, especially among LMICs. These countries should further apply this form of HTA in their healthcare decision-making, as it shows the efficiency of research prioritization and development processes, while ensuring that the needs of vulnerable populations are met.

10. That when making decisions about investments in STI initiatives, governments and other organizations explicitly consider likely trade-offs between SDGs, the distribution of benefits and costs, and the potential for unintended adverse effects. Prior to implementation, each initiative would be carefully assessed so that the full range of consequences is identified and evaluated. 
11. That considering how the desired scale of impact from STI initiatives for delivering SDG-related benefits is important. Strategies such as development of a theory of scaling can help in this by emphasizing the need for a clear focus on scaling, developing perspectives on how scaling occurs, considering how innovation characteristics affect scaling, and creating shared perspectives about scaling outcomes.

12. That STI strategies and policies focus on improving coordination, alignment, and collaboration with key STI stakeholders to identify challenges impeding development of an international STI framework that includes governments and businesses. There are technical, legal, trade, and institutional barriers that need attention to improve knowledge transfer, leverage of expertise and skills, and access to finance, at all stages of R\&D programs.

13. That to guide national investments, and to improve the likelihood of external funding support, STI initiatives be systematically prioritized, broken into smaller development packages, and presented to external donors with whose targets they align. Honing and clarifying these processes is important for building trust, assessing feasibility, establishing goals and timelines, and meeting milestones.

14. That STI policy in pursuit of sustainable agricultural production in developing regions address issues such as the balance between productivity and sustainability; the needs, constraints, and knowledge of resource-poor farmers; access to resources, markets, and infrastructure; the exercise of market power by input suppliers and output purchasers; effective agricultural extension that recognizes local conditions and farmer preferences; and efficient management of the supply chain beyond the farm gate.

\section{References}

Adenle, A. A., Azadi, H., and Arbiol, J. (2015). Global assessment of technological innovation for change adaptation and mitigation in developing world, Journal of Environmental Management 161,261-275.

Mazzucato, M. (2018). Mission-oriented innovation policies: challenges and opportunities, Industrial and Corporate Change 27(5), 803-815.

United Nations (2014). Science, technology and innovation for the post-2015 development agenda, Economic and Social Council. Report of the Secretary-General. Seventeenth session Geneva, May 12-16.

United Nations (2016). Science, technology, innovation and capacity-building: Chapter II.G. Addis Ababa Action Agenda-Monitoring commitments and actions. http://www. un.org/esa/ffd/wp-content/uploads/2016/03/2016-IATF-Chapter2G.pdf (accessed March 31, 2019).

World Bank (2008). Science, Technology, and Innovation: Capacity Building for Sustainable Growth and Poverty Reduction. The World Bank, Washington, DC. 